\title{
Acute organo-phosphorus pesticide poisoning in North Karnataka, India: oxidative damage, haemoglobin level and total leukocyte
}

*Hundekari IA ${ }^{\mathrm{I}}$, Suryakar $\mathrm{AN}^{2}$, Rathi $\mathrm{DB}^{\mathrm{I}}$

1. Department of Biochemistry, BLDEU's Shri B.M. Patil Medical College, Bijapur; Karnataka.Pin-586103

2. Registrar, Maharashtra University of Health Sciences, Nashik, Maharashtra, India.

\begin{abstract}
Background: Pesticide poisoning is an important cause of morbidity and mortality in India.

Objectives: To assess the oxidative damage, hemoglobin level and leukocyte count in acute organophosphorus pesticide poisoning.

Methods: Plasma cholinesterase was assessed as a toxicity marker. Oxidative damage was assessed by estimating serum malondialdehyde (MDA) levels, plasma total antioxidant capacity (TAC), erythrocyte superoxide dismutase (SOD), catalase (CAT) and glutathione peroxidase (GPx) levels.

Results: Progressive and significant decline $(\mathrm{p}<0.001)$ in plasma cholinesterase in correlation with the severity of organophosphorus poisoning was observed. Serum MDA levels significantly increased $(\mathrm{p}<0.001)$ in all grades of organophosphorus poisoning cases as compared to controls. Erythrocyte SOD, CAT and GPx were significantly increased $(\mathrm{p}<0.05)$ in earlier grade and $(\mathrm{p}<0.001)$ in later grades of organophosphorus poisoning cases as compared to controls. While plasma TAC $(\mathrm{p}<0.001)$ was significantly decreased in all grades of organophosphorus poisoning cases as compared to controls.

Leucocytosis observed in these cases signifies the activation of defense mechanism which could be a positive response for survival.

Conclusion: Organophosphorus compounds inhibit cholinesterase action leading to cholinergic hyperactivity. Increased MDA level may lead to peroxidative damages deteriorating the structural and functional integrity of neuronal membrane. Increased erythrocyte SOD, CAT and GPx activities suggest an adaptive measure to tackle the pesticide accumulation. Hence it is concluded that cholinesterase inhibition may initiate cellular dysfunction leading to acetylcholine induced oxidative damage.
\end{abstract}

Key words: Cholinesterase (ChE), leucocytosis, lipid peroxidation African Health Sciences 2013; 13(1): 129 - 136 http://dx.doi.org/10.4314/ahs.v13i1.18

\section{Introduction}

Organophosphorus (OP) pesticide poisoning is a major global health problem with thousands of deaths every year. Recent World Health Organization report shows that every year about three million cases of OP intoxications and 3,00,000 OP poisoning related death occurs worldwide ${ }^{1}$.

Organophosphorus compounds are phosphoric or phosphonic acid derivative which are irreversible inhibitors of both muscarinic and nicotinic acetyl cholinesterase (AChE) and affect the central nervous system. These compounds rapidly get absorbed following inhalation or ingestion; once

\footnotetext{
*Corresponding author:

Dr. Indira A. Hundekari

Department of Biochemistry

BLDEU's Shri B.M. Patil Medical College

Bijapur, Karnataka, India, 586103

Tel: $08352-262770$

E-mail: indira_hundekari@yahoo.com
}

absorbed the compounds get accumulated in fat, liver, kidneys and salivary glands ${ }^{2}$.

The primary mechanism of action of organophosphate pesticides is inhibition of carboxyl ester hydrolases, particularly acetyl cholinesterase (AChE, EC 3.1.1.7). AChE is an enzyme found on red blood cell (RBC) membranes that degrades the neurotransmitter acetylcholine $(\mathrm{ACh})$ into choline and acetic acid. ACh is found in the central and peripheral nervous system, neuromuscular junctions, and red blood cells. Pseudocholinesterase (BChE, EC 3.1.1.8), which is a liver acute phase protein present in bloodplasma and nervous tissue, is inhibited by organophosphorus compounds in a similar way to AChE, but the specificity of the two enzymes is different. Organophosphates inactivate AChE by phosphorylating the serine hydroxyl group located at the active site of AChE. The phosphorylation occurs by loss of an organophosphate leaving group and establishment of a covalent bond with AChE. 
Once AChE has been inactivated, ACh accumulates throughout the nervous system, resulting in over stimulation of muscarinic and nicotinic receptors. Clinical effects are manifested via activation of the autonomic and central nervous systems and at nicotinic receptors on skeletal muscle. BChE like other serine esterase reacts with OP compounds forming phosphorylated esterases ${ }^{3}$.

The leading cause of death in OP poisoning is respiratory failure. The mortality rate depends on the type of compound used, amount ingested, general health of the patient and delay in diagnosis or treatment. Patients with pesticide poisoning shows a wide spectrum of adverse health effects ranging from gastrointestinal symptoms to cardiac, immunological or neurotoxic diseases ${ }^{4}$.

Oxidative stress results when pro-oxidant are insufficiently balanced by antioxidants, resulting in cellular damage. Measurement of lipid peroxidation products, e.g. malondialdehyde (MDA) and endogenous oxygen free radical (OFR) scavengers such as SOD, CAT and GPx are effective markers to study OFR effects. Lipid peroxidation is increased in many disease states as well as in tissues poisoned by toxins. One of the molecular mechanisms of the toxicity of the pesticides seems to be lipid peroxidation; as a consequence these compounds can disturb the biochemical and physiological functions of red blood cells, hence the study was planned to assess the oxidative damage \& hematological parameters in acute organophosphorus poisoning.

\section{Methods}

The present study was carried out in the Department of Biochemistry; B.L.D.E.U's Shri B.M. Patil Medical College Hospital and Research Centre. The study protocol was approved by the institutional ethical committee and utmost care was taken during experimental procedure according to the Helsinki declaration. It includes 150 clinically diagnosed OP poisoning cases admitted in this hospital and 30 normal healthy control subjects. Diagnosis was based on information taken both from the patient or the patient's family and characteristic clinical signs and symptoms.

Clinically diagnosed OP poisoning cases on the basis of definite history by patient or attendants and also confirmed by clinical features and characteristic odour of stomach wash or vomitus were included in the study. Patients with double poisoning, chronic lung disease, pulmonary tuberculosis, renal failure, known neuromuscular disease, smokers and alcoholics which may interfere in assessment of oxidative stress; were excluded from the study.

The grouping of the OP poisoning cases was done depending upon signs and symptoms. Grade I - OP poisoned with no signs and symptoms $(\mathrm{n}=26)$.

Grade II - Diarrhea, vomiting, abdominal pain, giddiness $(\mathrm{n}=27)$.

Grade III - Pupillary constriction with above symptoms ( $\mathrm{n}=31)$.

Grade IV - Pulmonary edema $(\mathrm{n}=34)$.

Grade V - Unconsciousness ( $\mathrm{n}=32)$.

Immediately after admission to the hospital, before starting the appropriate treatment; $10 \mathrm{ml}$ venous blood samples were collected from the subjects under aseptic conditions. Out of this $4 \mathrm{ml}$ blood was collected in heparinised bulb and $6 \mathrm{ml}$ in plain bulb. Serum and plasma were separated by centrifugation at 3,000 rpm for 10 minutes, at room temperature. Then all samples were immediately placed at $40^{\circ} \mathrm{C}$ until they were processed to get accurate and reproducible results. After separation of the plasma, erythrocytes were washed three times with $0.9 \%$ saline solution. To one volume of washed erythrocytes nine volume of cold redistilled water was mixed then centrifuged for 10 minutes to get the hemolysate which was used for estimation of erythrocyte SOD, CAT and GPx.

Plasma ChE was estimated by Butyrylthiocholine Kinetic Method, using standard kit of Agappe Diagnostics. Cholinesterase act on butyrylthiocholine to form thiocholine which acts on dithio-bis-nitro benzoic acid giving pink colored 2-nitro, 5- mercaptobenzoate ${ }^{5,6}$.

Serum MDA concentration was measured as lipid peroxidation product by the Kei Satoh method. Serum proteins were precipitated by trichloro acetic acid (TCA) and the mixture was heated for 30 minutes with thiobarbituric acid in $2 \mathrm{M}$ sodium sulphate, in a boiling water bath. The resulting chromogen was extracted with n-butyl alcohol and the absorbance of the organic phase was determined at a wavelength of $530 \mathrm{~nm}$. The values were expressed in terms of $\mathrm{nmol} / \mathrm{ml}$ of malondialdehyde (MDA) using 1,1,3,3, tetra ethoxy propane as the standard ${ }^{7}$.

Activity of erythrocyte superoxide dismutase (SOD) was measured by the method of Marklund and Marklund. Superoxide anion is involved in the auto oxidation of pyrogallol at alkaline $\mathrm{pH}$ 8.5. The 
superoxide dismutase inhibits the auto-oxidation of pyrogallol, which can be determined as an increase in absorbance per two minutes at $420 \mathrm{~nm}$. The SOD activity was measured as Units/gms of $\mathrm{Hb}$. One unit of superoxide dismutase is defined as the amount of enzyme required to cause $50 \%$ inhibition of pyrogallol auto oxidation ${ }^{8}$.

Erythrocyte catalase was measured by the method of Aebi (1983). Heparinized blood was centrifuged and the plasma was removed. The erythrocytes were washed three times with $5 \mathrm{ml} \mathrm{0.9 \%}$ sodium chloride and lysed in 10 volumes of cold deionised water. The whole mixture was centrifuged further for $10 \mathrm{~min}$ at $3,000 \mathrm{rpm}$. The cell debris was removed and the clear hemolysate was diluted 500 times phosphate buffer $(60 \mathrm{mM}, \mathrm{pH}-7.4)$. Catalase decomposes hydrogen peroxide $\left(\mathrm{H}_{2} \mathrm{O}_{2}\right)$ to form water and molecular oxygen. In the ultra violet range, $\mathrm{H}_{2} \mathrm{O}_{2}$ shows a continual increase in absorbance with decreasing wavelength. At $240 \mathrm{~nm}, \mathrm{H}_{2} \mathrm{O}_{2}$ absorbs maximum light. When $\mathrm{H}_{2} \mathrm{O}_{2}$ is decomposed by catalase then the absorbance decreases. The decreased absorbance was measured at $240 \mathrm{~nm}$ at 15 second intervals up to $1 \mathrm{~min}$ and the difference in absorbance ( $\triangle A$ at $240 \mathrm{~nm}$ ) per unit time was measured. The unit of catalase activity was expressed as $\mathrm{mM}$ of $\mathrm{H}_{2} \mathrm{O}_{2}$ decomposed/mg Hb/min ?.

Erythrocyte Glutathione peroxidase (GPx) was assayed by Paglia and Valentine method. GPx catalyses the oxidation of glutathione by cumene hydroperoxide. In the presence of glutathione reductase and NADPH (reduced nicotinamide adenine dinucleotide phosphate) the oxidized glutathione is immediately converted to the reduced form with a concomitant oxidation of NADPH to $\mathrm{NADP}+$. The decrease in absorbance at $340 \mathrm{~nm}$ is measured ${ }^{10}$.

Table 1: Nature of poisoning cases in male and female

\begin{tabular}{lllc}
\hline Nature of poisoning & Male & Female & Total \\
\hline Suicidal & 86 & 57 & $143(95.33 \%)$ \\
Accidental & 5 & 1 & $6(4 \%)$ \\
Occupational & 1 & - & $1(0.67 \%)$ \\
& $92(61.33 \%)$ & $58(38.67 \%)$ & 150 \\
\hline
\end{tabular}

The plasma total antioxidant capacity (TAC) was estimated by FRAP (Ferric reducing ability of plasma) assay. The antioxidant power of plasma converts ferric ions to ferrous ions at low $\mathrm{pH}$ forming a pink coloured ferrous tripyridyl triazine $\left(\mathrm{Fe}^{\hat{\mathbf{E}}}-\right.$ TPTZ) complex. Ferrous reducing antioxidant power values were obtained by comparing the change in the absorbance at $593 \mathrm{~nm}$ in mixture with those of ferrous ion of known concentration. The TAC in plasma was expressed as $\mathrm{nmol} / \mathrm{ml}^{11}$. Hematological parameters were estimated using automated cell counter analyser; Sysmax Kx-21.

Statistical analysis of the data was performed using student $t$-test. The values were expressed as mean \pm standard deviation (Mean \pm SD). P values less than $0.05(\mathrm{p}<0.05)$ were considered as statistically significant.

\section{Results}

Organophosphorus compounds are highly toxic to human beings. Poisoning due to OP compounds is steadily increasing in India, probably because of the wide availability of pesticides as a result of extensive use in agriculture and also because of sale of these items over the counter.

In our study we found that the incidence of poisoning was more common among the age group between 15 to 35 years and males $86(57.33 \%)$ were more likely to attempt suicide than females 57 (38\%) (table 1).

Among the OP compounds the most commonly used were Dimethoate, Monocrotophos, Chlorpyrifos, Paraoxan, Mevinphos and Triazophos. 


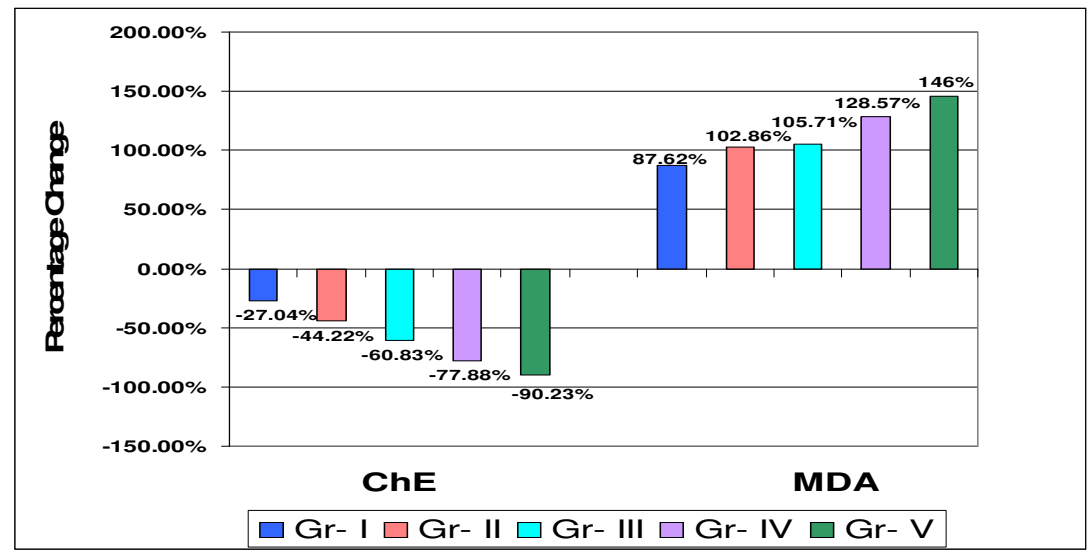

Figure 1: Percentage change graph of plasma $\mathrm{ChE}$ and serum MDA in all grades of OP poisoning cases with respect to control group

The plasma ChE level of normal healthy control group ranged from $4500-8000 \mathrm{U} / \mathrm{L}$ and in OP poisoning cases $400-4800 \mathrm{U} / \mathrm{L}$. We found significant $(\mathrm{p}<0.001)$ and progressive decline in plasma ChE levels in all grades of OP poisoning cases compared to controls. Further with an increase in the severity of poisoning there was corresponding decrease in plasma ChE activities as shown in table-2. Significant depression in plasma ChE activity suggests that estimation of plasma $\mathrm{ChE}$ is useful in confirmation of OP poisoning. The results are in consistent with earlier studies ${ }^{12}$. In earlier study we found a steady recovery in plasma $\mathrm{ChE}$ levels after treatment ${ }^{13}$. While no correlation between serum $\mathrm{ChE}$ level and severity of OP poisoning was also reported ${ }^{14}$.
Table 2: Mean values of Plasma Cholinesterase $(\mathrm{ChE})$ and Serum MDA levels in different grades of OP poisoning cases and control subjects:

\begin{tabular}{lcc}
\hline Study groups & $\begin{array}{l}\text { Plasma ChE } \\
(\mathbf{U} / \mathbf{L})\end{array}$ & $\begin{array}{l}\text { Serum MDA } \\
(\mathrm{nmol} / \mathrm{ml})\end{array}$ \\
\hline $\begin{array}{l}\text { Controls } \\
(\mathrm{n}=30)\end{array}$ & $6286 \pm 912.53$ & $1.05 \pm 0.37$ \\
$\begin{array}{l}\text { Grade I } \\
\text { (n=26) }\end{array}$ & $4586.4 \pm 259.9^{* *}$ & $1.97 \pm 0.39^{* *}$ \\
$\begin{array}{l}\text { Grade II } \\
\text { (n=27) }\end{array}$ & $3506 \pm 234.7^{* *}$ & $2.13 \pm 0.55^{* *}$ \\
$\begin{array}{l}\text { Grade III } \\
\text { (n=31) }\end{array}$ & $2462 \pm 239.2^{* *}$ & $2.16 \pm 0.52^{* *}$ \\
$\begin{array}{l}\text { Grade IV } \\
\text { (n=34) }\end{array}$ & $1390 \pm 254^{* *}$ & $2.39 \pm 0.61^{* *}$ \\
$\begin{array}{l}\text { Grade V } \\
\text { (n=32) }\end{array}$ & $614 \pm 211^{* *}$ & $2.52 \pm 0.64^{* *}$ \\
$\begin{array}{l}\text { Data presented are Mean } \pm \text { SD; } \\
\text { highly significant. }\end{array}$ & \\
\hline
\end{tabular}

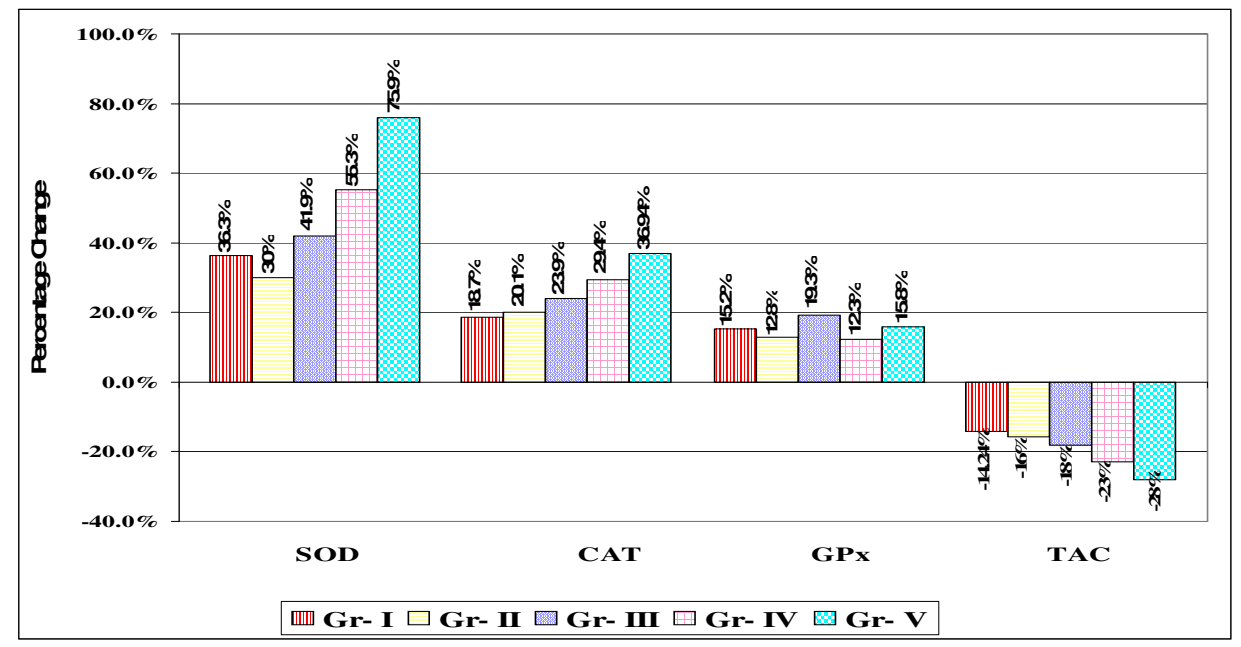

Figure 2 : Percentage change graph of mean levels of Erythrocyte superoxide dismutase (SOD), catalase (CAT), glutathione peroxidase (GPx) and plasma total antioxidant capacity (TAC) of organophosphorus (OP) poisoning cases with respect to control group 
The inhibition of ChE initiates the accumulation of free radicals leading to lipid peroxidation which may be the indicator of cell injury. The phospholipids component of biomembranes is believed to be the site of action of OP compounds ${ }^{15}$. The source of free radicals in acute OP poisoning is probably muscles. Free radicals play important role in toxicity of pesticides which may induce oxidative stress leading to generation of free radicals and alteration in antioxidants or oxygen free radical scavenging enzyme system ${ }^{16}$.

Significant $(\mathrm{p}<0.001)$ increase in serum MDA levels in all grades of OP poisoning cases compared to controls was observed. The magnitude of increase in MDA was related with severity of $\mathrm{OP}$ poisoning. Toxic manifestations induced by OP compounds may be associated with enhanced production of reactive oxygen species (ROS) which induces the oxidative process and lipid peroxidative damage in cell membranes. ROS are implicated as important pathologic mediators in many disorders. Increased generation of ROS and enhanced lipid peroxidation are considered responsible for the toxicity due to pesticides ${ }^{17}$.

Many intrinsic radical scavenger systems involve enzymatic and nonenzymatic reactions. Significantly increased $(\mathrm{p}<0.05)$ activities of erythrocyte superoxide dismutase (SOD) in grade Ist and IInd and $(\mathrm{p}<0.001)$ in grade IIIrd, IVth, Vth of organophosphorus poisoning cases as compared to controls was observed. While erythrocyte catalase (CAT) significantly increased $(\mathrm{p}<0.05)$ in Ist to IVth grades of organophosphorus poisoning cases with significant $(\mathrm{p}<0.001)$ increase in $\mathrm{Vth}$ grade as compared to controls. Significant $(p<0.05)$ increase in erythrocyte glutathione peroxidase (GPx) levels in Ist and IInd grade while $(\mathrm{p}<0.001)$ in IIIrd, IVth and $V$ th grades organophosphorus poisoning cases as compared to controls was also observed. SOD, CAT and GPx are important components of enzymatic antioxidative systems. Increase in SOD activities in erythrocytes of OP poisoning cases indicates an increased production of superoxide radical. Increased CAT activities in erythrocytes may be explained by their influence on hydrogen peroxide as substrate, which is formed in the process of dismutation of superoxide radicals. GPx is a selenium dependent enzyme which removes both $\mathrm{H} 2 \mathrm{O} 2$ and lipid peroxide by catalyzing the conversion of lipid hydroperoxides to hydroxyl acids in presence of reduced glutathione. GPx catalyses the reduction of hydroperoxides using GSH, thereby protecting mammalian cells against oxidative damage. Glutathione and glutathione dependent enzyme systems provide major protection against toxic agents $^{18}$.

Generally there is an inverse relationship between lipid peroxidation and antioxidant enzymes; however we found significant increase in erythrocyte SOD, CAT and and GPx activities (table no. 3) as well as serum MDA concentration. Erythrocyte SOD, CAT and GPx efficiently scavenges toxic free radicals and are partly responsible for protection against lipid peroxidation due to acute/chronic organophosphorus pesticide exposure. Thus the increase in these enzymes was probably a response towards increased ROS generation in OP toxicity. Supporting our results there is evidence that administration of malathion resulted in increased SOD, CAT as well as MDA concentration in RBCs and liver of rats ${ }^{15}$.

Table 3: Mean values of enzyme antioxidant (SOD, CAT and GPx) levels and plasma TAC in different grades of OP poisoning cases and control subjects:

\begin{tabular}{|c|c|c|c|c|}
\hline Study groups & $\begin{array}{l}\text { Erythrocyte } \\
\text { SOD } \\
\text { (Units / gms } \\
\text { of } \mathrm{Hb} \text { ) }\end{array}$ & $\begin{array}{l}\text { Erythrocyte } \\
\text { CAT (mM H} \mathrm{H}_{2} \\
\text { decomposed/ } \\
\mathrm{mg} \mathrm{Hb} / \mathrm{min})\end{array}$ & $\begin{array}{l}\text { Erythrocyte } \\
\text { GPx (U/L) }\end{array}$ & $\begin{array}{c}\text { Plasma TAC } \\
(\mathrm{nmol} / \mathrm{ml})\end{array}$ \\
\hline Controls $(\mathrm{n}=30)$ & $10.14 \pm 3.6$ & $15.54 \pm 5.67$ & $5351 \pm 935$ & $1264 \pm 130$ \\
\hline Grade I $(n=26)$ & $13.82 \pm 7.26 *$ & $18.44 \pm 6.54 *$ & $6164 \pm 1267 *$ & $1084.7 \pm 173 * *$ \\
\hline Grade II $(n=27)$ & $13.19 \pm 5.54 *$ & $18.67 \pm 7.03 *$ & $6035 \pm 1210 *$ & $1066.8 \pm 122 * *$ \\
\hline Grade III $(n=31)$ & $14.39 \pm 5.14 * *$ & $19.25 \pm 6.98^{*}$ & $6384 \pm 1378^{* *}$ & $1040 \pm 140 * *$ \\
\hline Grade IV $(\mathrm{n}=34)$ & $15.75 \pm 6.0 * *$ & $20.11 \pm 7.9 *$ & $6007 \pm 1355^{* *}$ & $975.5 \pm 198 * *$ \\
\hline Grade V $(n=32)$ & $17.84 \pm 7.54 * *$ & $* 21.28 \pm 7.68 * *$ & $6197 \pm 1353 * *$ & $912.6 \pm 162 * *$ \\
\hline
\end{tabular}




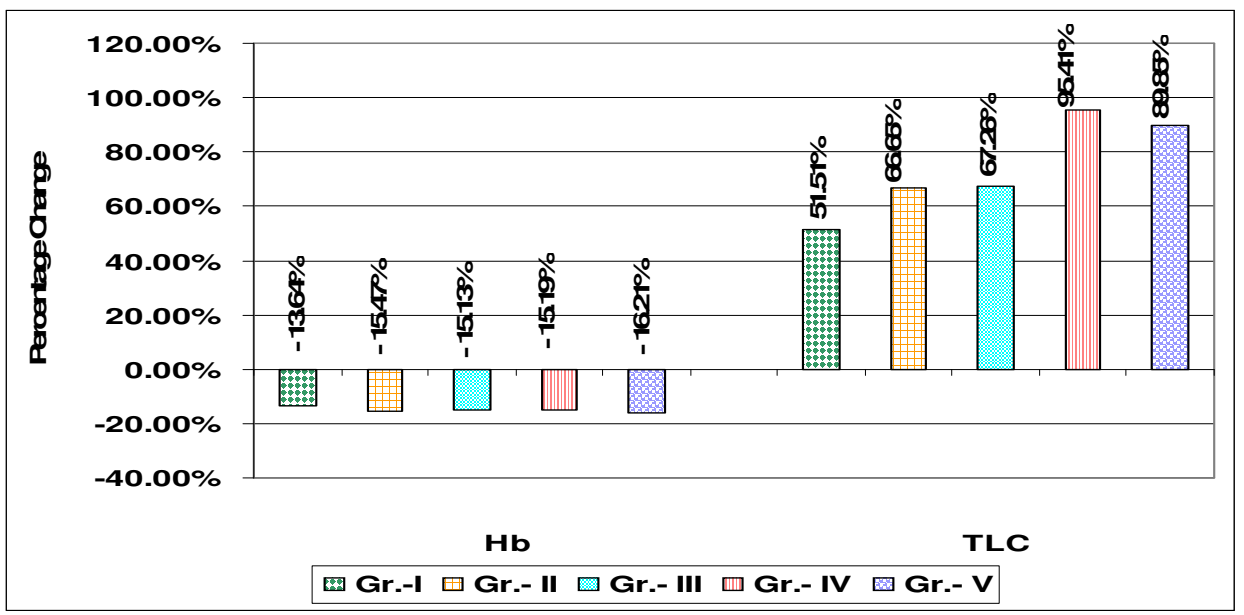

Figure 3: Percentage change graph of mean levels of $\mathrm{Hb}$ concentration and Total Leucocyte Count in all grades of organophosphorus (OP) poisoning cases with respect to control group

Table 4: Mean values of Hemoglobin concentration and total leucocyte count in OP poisoning cases and control subjects:

\begin{tabular}{llr}
\hline Study groups & Hb $(\mathbf{g m} \%)$ & $\begin{array}{r}\text { Total Leucocyte } \\
\text { Count }\end{array}$ \\
\hline Controls $(\mathrm{n}=30)$ & $14.74 \pm 2.98$ & $7735 \pm 2980$ \\
Grade I $(\mathrm{n}=26)$ & $12.73 \pm 1.84^{\bullet}$ & $11719 \pm 950 * *$ \\
Grade II $(\mathrm{n}=27)$ & $12.46 \pm 1.2^{*}$ & $12891 \pm 1105 * *$ \\
Grade III $(\mathrm{n}=31)$ & $12.51 \pm 1.09 *$ & $12938 \pm 2025^{* *}$ \\
Grade IV $(\mathrm{n}=34)$ & $12.50 \pm 0.95^{*}$ & $15115 \pm 1915^{* *}$ \\
Grade V $(\mathrm{n}=32)$ & $12.35 \pm 1.07 *$ & $14685 \pm 2245^{* *}$ \\
\hline
\end{tabular}

Data presented are Mean \pm SD; Note: $*$ indicates $\mathrm{P}<0.05-$ significant, $* *$ indicates $\mathrm{p}<0.001$ - highly significant, ${ }^{\bullet}$ indicates $\mathrm{p}>0.05-$ non significant.

\section{Discussion}

The study showed significant decrease $(p<0.001)$ in plasma total antioxidant capacity (TAC) in all grades of OP poisoning as compared to controls. The human body has several mechanisms to counteract with the damage produced by free radicals; the basic and the most prominent defense mechanism of human body are antioxidant agents. The overall total antioxidant capacity considers the cumulative effect of all antioxidant (known and unknown, measurable and nonmeasurable, enzymatic and nonenzymatic) present in plasma or serum of humans. Thus in acute OP poisoning sudden overproduction of reactive oxygen species leads to significant lipid peroxidation. The depletion of total antioxidant capacity induced by oxidative stress is eliminated by release of stock organ antioxidants, mainly from liver and adipose tissue and the induction or activation of antioxidant enzymes or the nonenzymatic antioxidants might be utilized to counteract the damage produced by free radicals. Due to consumption of nonenzymatic antioxidant agents which body could not compensate in a short period; hence the patient showed lower total antioxidant capacity. Ranjbar et al. reported significant lipid peroxidation accompanied with decreased TAC and ChE activity ${ }^{19}$.

The susceptibility of erythrocytes and lymphocytes to oxidative stress due to pesticide exposure is a function of overall balance between degree of oxidative stress and antioxidant defense capability. Thus the OP compounds may directly or indirectly modify the antioxidant defense capability of exposed subjects and thus affect their susceptibility to oxidative stress ${ }^{16}$.

The present findings indicate that cells continually suffer from oxidative stress in spite of over activity of antioxidant defense mechanism as 
indicated by increase in erythrocyte SOD, CAT and GPx activity. The severity of OP poisoning shows more stress and hence more free radical generation. The free radical production is so high that it even overwhelms the elevated antioxidant failing to check lipid peroxidation. The higher levels of antioxidant enzymes may be necessary to detoxify increased concentration of lipid peroxidation products that are generated from oxidative stress due to OP toxicity. We also observed that the white blood cell count significantly $(p<0.001)$ increased in all grades of OP poisoning cases (table 4 and figure 3 ). We found leucocytosis with mild neutrophilia as compared to controls. The results indicated significant decrease in the mean value of hemoglobin in the OP poisoning group compared to the control group possibly as a result of the decreased synthesis of hemoglobin concentration. Another possible interpretation is binding of organophosphorus insecticides on iron, followed by a lack of incorporation of iron in hemoglobin leading to decreased size of red blood cells. However, the significantly higher leukocytes count observed in the OP poisoning signifies the activation of the defense mechanism and immune system, which could be a positive response for survival ${ }^{20}$.

\section{Conclusion}

Thus the present study revealed that estimation of plasma ChE determines the severity of poisoning which can be helpful for predicting outcome in OP poisoning. Toxicities of OP compounds induce oxidative damage by elevating lipid peroxide levels. Increased antioxidant enzymes may be attributed to adaptive response of erythrocytes to oxidative damage due to OP compounds. This study will be helpful in management and follow up of OP poisoning related oxidative damage.

\section{References}

1. Eddleston M, Phillips MR. Self poisoning with pesticides. BMJ 2004; 328:42-44.

2. Vale JA. Toxicokinetic and toxicodynamic aspects of organophosphorus insecticide poisoning. Toxicol Lett 1998; 102:649-652.

3. Reiner E., Radic Zoran and Vera SimeonRudolf. Mechanisms of organophosphorus toxicity and detoxication with emphasis on studies in Croatia. Arb Hig Rada Toksikol 2007; 58:329-338.
4. Shahin Shadnia, Ebrahim Azizi, Rohollah Hosseini, Samideh. Evaluation of oxidative stress and genotoxicity in organophosphorus insecticide formulators. Hum Exp Toxicol 2005; 24, 439-445.

5. Kendel M, Bottger R. A kinetic method for determination of the activity of pseudocholinesterase. Klin Wochenscher 1967; 45:325.

6. Norber Tietz, editor. Textbook of Clinical Chemistry. 3rd ed. W.B. Saunders Company, Philadelphia; 1986.

7. Kei Satho. Serum lipid peroxide in cerebrovascular disorders determined by a new colorimetric method. Clin Chimica Acta 1978; 90:37-43.

8. Marklund and Marklund (1974) modified by Nandi et al Assay of SOD activity in tissue. $J$ Biochem 1988; 13(3):305-15.

9. Aebi H. Catalase in vitro. Methods Ensymol 1984; 105:121-26.

10. Paglia D. E. and Valentine. J Lab Clin Med 1967; 70:158.

11. Iris F, Benzi F, Strain S. Ferric reducing antioxidant assay. Methods Enaymol 1999; 292:15-27.

12. M. Dandapani, A Zachariah, MR Kavitha, L Jeyaseelan and A Oomen. Oxidative damage in intermediate syndrome of acute organophosphorus poisoning. Ind J Med Res 2003:253-259.

13. Hundekari, I Suryakar AN, Rathi DB. Oxidative stress and antioxidant status in acute organophosphorus pesticide poisoning cases of North Karnataka India. J Env Hlth Res 2011;11:39-44.

14. Cherian MA, Roshini C, Visalakshi J, Jeyaseelan L, Cherian A M. Biochemical and clinical profile after acute organophosphorus poisoning - A placebo controlled trial using PAM. JAPI 2005; 53:427-431.

15. Akhgari M, Abdollahi M, Kebryaeezadeh A, Hosseini R, Sabzevari O. Biochemical evidence for free radical induced lipid peroxidation as a mechanism for subchronic toxicity of malathion in blood and liver of rats. Hum Exp Toxicol 2003; 22: 205-211.

16. Banerjee BD, Seth V, Bhattacharya A, Pasha ST, Chakraborty AK. Biochemical effects of some pesticides on lipid peroxidation and free radical scavengers. Toxicol Lett 1999; 107:33-47.

17. Halliwell and Gutteridge JMC. Oxygen free radicals and iron in relation to biology and 
medicine: some problem and concepts. Arch Biochem Biophys 1986; 246:501- 514.

18. Avinash Tope, Frederick N. Bebe, Myna Panemangalore. Micronuclei frequency in lymphocytes and antioxidants in the blood of traditional limited resource farm workers exposed to pesticides. J Env Sci and Hlth 2006; 41 (pt-B):843.
19. Ranjbar A, Solhi H, Mashayekhi FJ, Susanabdi A, Rezaie A, Abdollahi M. Oxidative stress in acute human poisoning with organophosphorus insecticides: a case control study. Env Toxicol Pharmacol 2005; 20:88-91.

20. Wesseling C, McConnell R, Partanen T. Agricultural pesticide use in developing countries: Health effects and research needs. Int J Hlth Res 1997; 27: 273-308 